\section{Und es geht noch mehr}

Ursache für diese Kostendegression sei, dass sich die Zahl der Praxen reduziert habe und gleichzeitig die Zahl der erbrachten Leistungen gestiegen sei, so Wölbing. Wölbing schließt daraus, dass die Ärzte durchaus noch freie Kapazitäten haben. „Dass wir beobachten, dass sich Sprechzeiten verkürzen oder es schwerer wird einen Arzttermin zu erhalten, hat ja nicht unbedingt etwas mit einer Überlastung der Ärzte zu tun“, sagte er.

Das Zi hat betriebswirtschaftlich gesehen hier den besseren Ansatz - auch, wenn die Krankenkassen das nicht wahrhaben wollen. Denn es unterteilt seine Studie in 29 Fachgruppen und weist für diese Einzelergebnisse auf. Sicher, auch das Zi bildet einen Mittelwert für alle Praxen. Der zeigt, dass das kalkulatorische Arzteinkommen aus GKVTätigkeit bei einer Normarbeitszeit von 51 Wochenstunden im Jahr 2008 bei $92.000 €$ lag. „Das sind $13 \%$ weniger als der vom Bewertungsausschuss festgesetzte Referenzwert von 105.571,80 €“, so von Stillfried. Aber das Institut hat die Daten der Vertragsärzte je nach Fachgruppenverteilung im Bundesarztregister und Regionstyp gewichtet. Denn das zeigt das ZiPP ganz klar: Eine Stadtpraxis hat andere Kostensätze und nicht selten sogar niedrigere GKV-Umsätze als eine Landarztpraxis. Dafür sind die Arbeitszeiten für den Arzt auf dem Land aber in der Regel höher als in der Stadt.

\section{Schwachpunkte haben beide Statistiken}

Egal ob die Krankenkassen es hören wollen oder nicht, die Arbeitszeit gehört mit in die Kostenrechnung, denn die Ärzte sind nun einmal diejenigen, die den Hauptanteil der reinen Patientenversorgung in den Praxen stemmen. Aber: Schwachpunkte weisen beide Statistiken auf. Denn die Daten vom ZiPP beruhen auf gerade einmal rund 4.600 Antwortbögen. Und: Will man genau rechnen, müsste man auf der Kostenseite ebenfalls den Anteil der privaten Krankenversicherung herausrechnen. Rebekka Höhl

\title{
Neue GOÄ kommt frühestens nach der Bundestagswahl
}

n dieser Legislaturperiode wird es keine neue Gebührenordnung für Ärzte (GÖ̈) mehr geben. Das hat Dr. Bernhard Rochell, Hauptgeschäftsführer der Bundesärztekammer (BÄK), beim 4 . Bundeskongress Privatmedizin in Köln schon bestätigt. Zwischen dem PKVVerband und der BÄK gebe es auch nach der ergebnislosen Klausurtagung über die Ausgestaltung der neuen GOÄ weiter Gespräche. Es seien noch „vielfältige Beratungsthemen“ vorhanden, so Rochell. Ein gemeinsamer Vorschlag von $\mathrm{BÄK}$ und PKV-Verband sei nicht in Sicht. Laut Rochell werde die BÄK im Falle des Falles einen eigenen Weg gehen und ein Modell für die neue GOÄ vorlegen, damit es vorangeht. Das könnte jetzt im Frühjahr so weit sein.

Eine „EBMisierung“ der GOÄ werde es mit der BÄK nicht geben, betonte Rochell. Bei einer zu starken Annäherung der beiden Gebührenordnungen „stellt sich die Frage nach der Existenzberechtigung der PKV“. „Auch der Gebührenrahmen muss bestehen bleiben, es ist nur die Frage der Spannbreite bei den Faktoren“, so der BÄK-Hauptgeschäftsführer weiter. Diese werde möglicherweise geringer. „Aber dann muss es auch eine realistische Basisvergütung geben, damit man nicht erst mit 2,3-fachem Faktor auf eine angemessene Vergütung kommt.“

\section{Analogbewertungen sollen die} Abrechnung weiterhin erschweren „Analogbewertungen machen das Leben mit der guten alten Tante GOÄ zunehmend schwer", beschrieb Rochell den aktuellen Zustand mit der Gebührenordnung. Mit der neuen GOÄ müsse es das auch weiterhin geben, allerdings weniger als heute, da Innovationen regelmäßig in die GOÄ aufgenommen werden sollen, führte der BÄK-Hauptgeschäftsführer aus.
Schluss mit arztindividuell kodierten BtM-Rezepten

Arztindividuell kodierte Betäubungsmittelrezepte wird es bald nicht mehr geben. Die Bundesopiumstelle im Bundesinstitut für Arzneimittel und Medizinprodukte (BfArM) wird Anfang 2013 beginnen, neue Rezeptformulare auszugeben, die mit etlichen Sicherheitsmerkmalen versehen sind. Der jetzige Kodierprozess sei zu zeitaufwendig. Künftig erfolgt die Zuordnung des einzelnen Rezeptes zum verordnenden Arzt über eine neunstellige Rezeptnummer. Alte BtM-Rezeptformulare sollten aber nicht an die Bundesopiumstelle zurückgeschickt, sondern vollständig aufgebraucht werden, so das BfArM.

$\mathrm{Cw}$

\section{Ärzte in Telemedizin schulen}

Telemedizinische Anwendungen werden für Ärzte immer wichtiger. Experten fordern nun, die Telemedizin in der ärztlichen Qualifikation zu verankern. In welchen Themenbereichen eine Fortbildung notwendig ist, hat die Bundesärztekammer in einem Workshop mit relevanten Fachgesellschaften und den Fortbildungsbeauftragten der Landesärztekammern erarbeitet. Das Ergebnis: Durch das heterogene Anwendungsspektrum telemedizinischer Methoden dominieren gebietsspezifische Anforderungen. Ein fachübergreifendes Fortbildungskonzept bietet sich nicht an.

wul

\section{Rezepturen: Gesprächsbedarf} nimmt zu

Die im Juni letzten Jahres in Kraft getretene neue Apothekenbetriebsordnung zwingt Ärzte und Apotheker dazu, mehr miteinander zu reden. Grund: Für individuelle Rezepturen ist Apothekern nun vorgeschrieben, eine Herstellungsanweisung zu erstellen, eine Plausibilitätsprüfung vorzunehmen und das Ergebnis zu dokumentieren. Bedenken muss der Apotheker mit dem verschreibenden Arzt klären. „Um eine reibungslose Belieferung der Rezepturen sicherzustellen, sollten Ärzte möglichst eine standardisierte Rezeptur verordnen, zum Beispiel aus dem ,Neuen Rezeptur-Formularium" “ (NRF), empfiehlt Dr. Peter Potthoff, Vorstandschef der KV Nordrhein. 